\title{
Evaluation and Control Strategy of the Regulation Capacity of Electric Heating Load
}

\author{
Yuxing $\mathrm{Li}^{1}$, Yu Shi ${ }^{1}$, Hao $\mathrm{Li}^{1}$, Xuefeng $\mathrm{Gao}^{1}$, Yeyang $\mathrm{Zhu}^{2, *}$ \\ ${ }^{1}$ State Grid Economic And Technical Research Institute Of Jilin Electric Power., LTD \\ ${ }^{2}$ University of Southern California
}

\begin{abstract}
In face of increasingly severe environmental problems, green and low-carbon power supply has become an important trend of current development. At present, the smart grid which will respond to the demand has improved the interaction between current users and the grid. In addition, electric heating and solar power generation and other renewable power generation methods are promoted in north China. Electric heating is a flexible and regulatable load; when it reaches a certain scale, it is bound to become an objective demand response resource in power grid operation, so it is of great practical significance to explore the evaluation of the regulation capacity of electric heating load.
\end{abstract}

\section{Electric Heating Load Modeling and Regulation Capacity Evaluation Analysis}

\subsection{Electric Heating Modeling}

\subsubsection{Dynamic Model of Electric Heating Load}

Electric heating load modeling is based on the demand response of electric heating, which includes architecture thermodynamics and electrothermal conversion theory. These two theories are mainly used to discuss the relationship between the indoor temperature and the heat supply, and establish the relationship between heat supply and power according to their relationship. ${ }^{[1]}$ The application of the two theories can consider the relationship between the indoor temperature and power of the building, and the structure of the enclosure of buildings in architectural components. In order to improve thermal insulation, the enclosure structure function of buildings is gradually enhanced, and the heat storage capacity is strong. The interior of the building will not be affected by the external environment temperature, which indicates that the thermal inertia of the building increases.

According to the principle of heat balance, when the indoor heat supply of the building exceeds the heat dissipation of the building enclosure structure, part of the heat will accumulate in the building, leading to a rise in the internal temperature of the building. ${ }^{[2-4]}$ On the contrary, the heat will be further released, and the indoor temperature of the building will drop. When the two values are equal, the building will reach the balance state, and the indoor temperature will not change in a short period of time.
According to the analyses above, the energy transfer process between the environment and the building can be assessed. By analyzing the temperature differences among the indoor air, external wall and internal wall of the building, we can build the equation between the temperature and variable parameters in the working state:

$$
\mathrm{Q}=\frac{\sum \mathrm{Q}_{\mathrm{i}}}{\mathrm{A}}=\frac{\sum \varepsilon \cdot \mathrm{K} \cdot \mathrm{S}\left(\mathrm{T}_{\text {out }}(\mathrm{t})-\mathrm{T}_{\text {in }}(\mathrm{t})\right)}{\mathrm{A}}
$$

In Eq. (1), Q is the transferred heat per unit building area in one unit time, and $\mathrm{A}$ and $\mathrm{S}$ represent the total area and exterior area of standard users determined by building shape coefficient, respectively.

According to the law of conservation of energy, the energy change value of electric heating standard user and the difference between electric heating heat and building heat in different periods of time are fully considered to construct the energy conservation model, and the formula of the current indoor temperature change is deduced:

$$
\frac{\mathrm{dTi}}{\mathrm{dt}}=\alpha\left(\mathrm{T}_{\text {in }}(\mathrm{t})-\mathrm{T}_{\text {out }}(t)\right)+\beta-\delta Q
$$

The coefficients of $\alpha, \beta, \delta$ in Eq. (2) are constants.

If the difference between the exterior wall and the interior wall of the building is ignored, it can be assumed that the indoor air temperature and the solid temperature of the building are equal, then we can have the following formula according to the heat balance equation:

$$
\begin{aligned}
& \left.\mathrm{T}_{\text {in }}(\mathrm{t})=\exp \left(-\frac{\mathrm{KS}}{\mathrm{C}_{\text {air }} \rho_{\text {ar }} \mathrm{V}} \Delta \mathrm{t}\right) \cdot\left(\mathrm{T}_{\text {in }}(\mathrm{t}-1)\right)-\mathrm{T}_{\text {out }}(\mathrm{t})-\mathrm{P}_{\text {heat }}(\mathrm{t}) \frac{1}{\mathrm{KS}}\right) \\
& +\left(\mathrm{T}_{\text {out }}(\mathrm{t})+\mathrm{P}_{\text {heat }}(\mathrm{t}) \frac{1}{\mathrm{KS}}\right)
\end{aligned}
$$

\footnotetext{
${ }^{*}$ Corresponding author: yeyangzh@usc.edu
} 


\subsubsection{Establishment of a Human Thermal Comfort Model}

According to the Code for Design of Heating, Ventilation and Air Conditioning, the temperature range of thermal comfort in winter in cold areas is $18{ }^{\circ} \mathrm{C}$ $22{ }^{\circ} \mathrm{C}$. At present, central heating is used in heating period. Through the optimization and control of electric heating, the temperature in buildings is kept within the comfortable temperature range of the human body. ${ }^{[5-7]}$ The power of electric heating is changed to realize the regulation of the initial temperature. The thermal demand of the human body is an important index to maintain the indoor temperature, so the electric heating system needs to provide enough heat to meet the thermal comfort of human body. The thermal comfort will be affected by many factors, such as psychology, behavior and environment. Therefore, the thermal comfort is a range, not a certain value. Here, the thermal comfort model is built based on fuzzy mathematics.

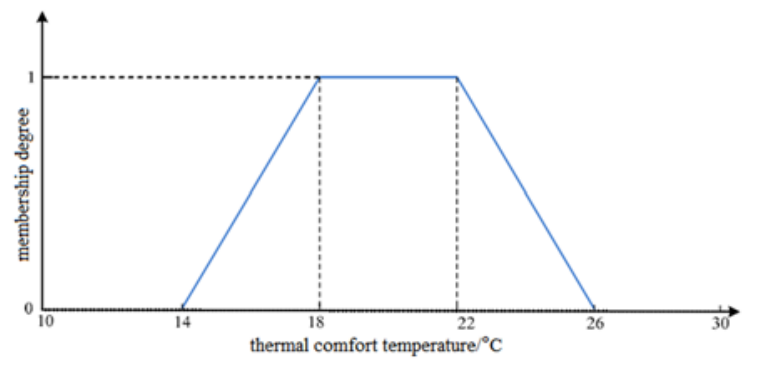

Fig. 1. Membership Degree Function Curve of Human Thermal Comfort Temperature

The trapezoidal membership degree function is used to show the comfort degree during the heating period. When the membership degree value is set as 1 , the temperature range can be used as the upper and lower limits of the thermal comfort range, and it can be used to constrain the indoor temperature value, and thus we obtain:

$$
\mathrm{T}_{\text {down }}<\mathrm{T}_{\text {in }(\mathrm{t})}<\mathrm{T}_{\text {up }}
$$

\subsection{Evaluation of Load Control Capacity of User Electric Heating Based on Temperature Prediction}

\subsubsection{Index of Load Regulation Capacity}

The process of electric heating is a temperature control device which transforms clean electric energy into heat energy, which is mainly used to determine the control space according to the temperature control range. Therefore, the regulation capacity of electric heating load can be defined as:

(1) Load control power of electric heating $P_{\text {control }}$

(2) The duration time $t_{\text {control }}$ of maintaining electric heating load power $\mathrm{P}_{\text {control }}$

(3) Controlled electricity quantity within power control time $\mathrm{W}_{\text {control }}$

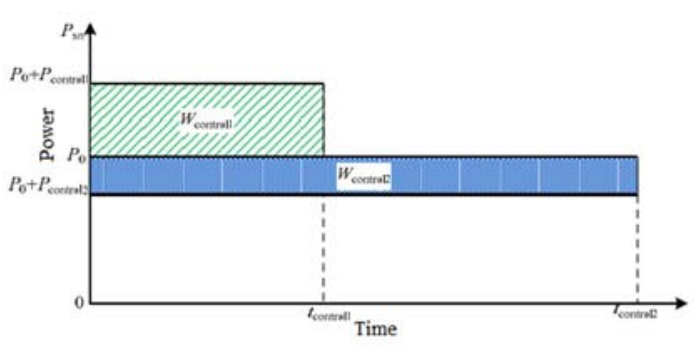

Fig. 2. Schematic Diagram of Controlled Electricity Quantity and Its Duration Changing with Power Control

\subsubsection{Evaluation Method of Electric Heating Load Control Capacity}

In evaluation of the load regulation ability of user electric heating, firstly, the temperature value of the weather in a range of time should be introduced according to the index. According to the previous dynamic model of electric heating, it is used to evaluate the load regulation ability of electric heating within the range of human body comfort. The specific process is shown in Figure 3. In order to clearly explain the content of this paper, we especially introduce parameters of $T_{1}$, $T_{2}, \ldots T_{k}$ as the temperature series obtained by weather forecast in a certain period of time in the future, in which the initial temperature is taken as $\mathrm{T}_{0}, \mathrm{P}_{0}$ is the power to maintain the indoor temperature $\mathrm{T}_{0}$, the maximum electric heating power value $\mathrm{P}_{\mathrm{sn}}$ and the most comfortable range $\left[T_{\text {down }}, T_{\text {up }}\right]$ of indoor building temperature under this temperature state.

(1) Input the initial value, and the output value includes the external surface area, comprehensive thermal conductivity coefficient of the surface and space volume of the user building. According to the temperature series parameters obtained from the weather forecast, the external temperature is finally determined as $T_{\text {out }}$. During the evaluation period, the initial temperature, local air specific heat capacity and air density in the whole warm season are determined;

(2) The parameters above are brought into a thermodynamic equivalent parameter model of electric heating for actual users. In this model, the indoor temperature change rate is 0 , and the electric heating power required for the obtained indoor temperature is $\mathrm{P}_{0}$;

(3) Evaluate the regulatable index control range of user electric heating load, in which the positive and negative regulatable ranges of heating power are $\left[0, \mathrm{P}_{\mathrm{sn}^{-}}\right.$ $\left.\mathrm{P}_{0}\right]$ and $\left[-\mathrm{p}_{0}, 0\right]$ respectively.

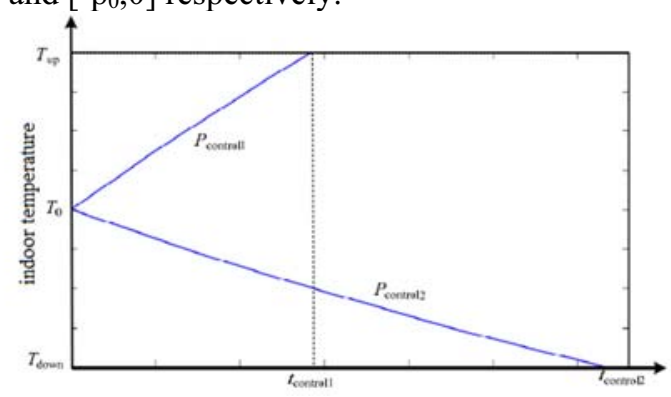

Fig. 3. Variation of Indoor Temperature with Time During Power Control 
(4) According to Figure 3, the control range can be evaluated, and the assessment can be carried out according to the duration of given controlled power. In the process, the thermal dynamic model needs to be used for calculation and analysis. Here, it can be divided into two situations for discussion. For example, when the value of control power exceeds 0 , the value of indoor temperature Tin will increase continuously, and when the temperature reaches the upper limit value $\mathrm{T}_{\text {up }}$, it will stop. If the regulatable power is lower than 0 , the indoor temperature of the building will decrease continuously. It will stop when the indoor temperature reaches the lower limit of Tdown. Therefore, the evaluation index of the user electric heating load regulation ability can be determined according to Figure 3.

(5) According to Figure 3, when the power control lasts for a period of time, the evaluation of $\mathrm{P}_{\text {control }}$ and $\mathrm{T}_{\text {control }}$ can be realized, and according to the specific data, it can be brought into the formula to evaluate the electricity quantity $\mathrm{W}_{\text {control }}$ that can be controlled during the power control period.

$$
\mathrm{W}_{\text {control }}=\int_{0}^{t_{\text {control }}} \mathrm{P}_{\text {control }}(t) d t
$$

\subsubsection{Influencing Factors of Electric Heating Load Control Capacity}

According to the transient equilibrium relation model of electric heating, many factors need to be considered in the evaluation of load regulation capacity, such as the randomness of thermodynamic parameters. According to the comprehensive thermal conductivity of the external surface of buildings, the specific heat capacity and density of the air in the heating area at a certain time, the evaluation capacity will be affected for the above parameters have randomness; secondly, it is the temperature range of thermal comfort. The electric heating load mainly controls and maintains the indoor temperature according to the power, so the selection of temperature should meet the requirement of human body comfort, which is also an important factor affecting the electric heating control. For the comfort degree is a range, in this paper, based on fuzzy mathematics, the range of temperature when the membership degree is set at 1 is set as the upper limit and the lower limit; the set value of indoor temperature can only be analyzed by the heat balance equation when ignoring the difference between the exterior wall and the interior wall of the building; if we want to obtain the outdoor temperature in a certain period of time, its change rate will affect the indoor temperature, and then affect the duration of power control, so as to interfere with the control of electric energy. To sum up, the outdoor temperature will affect the power consumption range of electric heating.

\section{MODELING AND CONTROL ANALYSIS OF ELECTRIC HEATING LOAD CLUSTER}

\subsection{Cluster Aggregation Model of Electric Heating Load}

According to the previous evaluation of electric heating load control capacity, it is found that the electric heating load can be used as demand response resource, and it is more suitable for cold regions such as northern China. In demand response control, it is necessary to realize cluster aggregation of electric heating load through a certain method, and it will not affect the power system dispatching in the meantime. ${ }^{[8-10]}$ The dispatching process mechanism is shown in Figure 4.

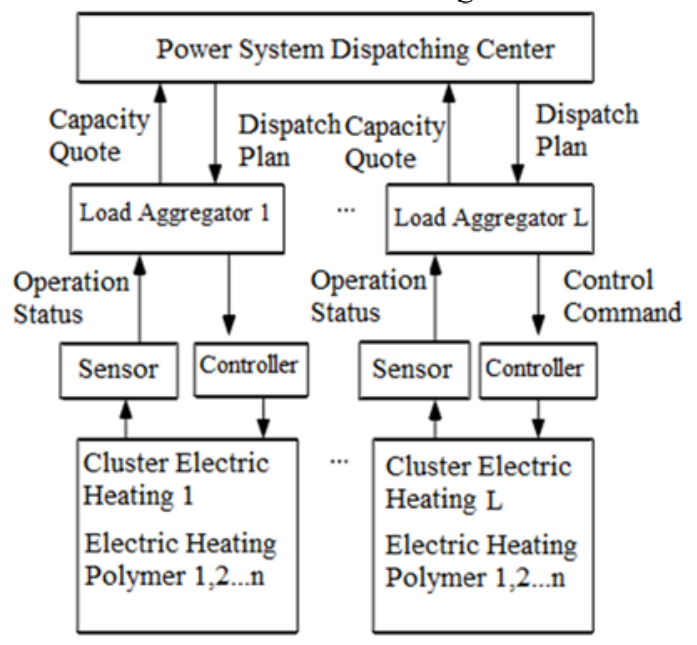

Fig. 4. Flow Chart of Cluster Electric Heating Dispatching Mechanism

According to the actual demand, an intermediary between the dispatching center and the electric heating users is required. The intermediary is the load aggregator, which is mainly used to unify the scattered electric heating loads and participate in the cluster power grid control. In advanced power grids and developed communication technology, the load aggregator can explore the load control capacity of the electric heating users and achieve the effect of balancing the power demand.

Figure 4 shows the cluster dispatching mechanism of electric heating loads. The load aggregator controls and manages the multi-electric heating load, and controls the whole operation state of the load group. It will issue the power command at the peak of power consumption or low power demand, and achieves the purpose of centralized control. As Figure 4 shows, the power system dispatching center mainly refers to the load forecast and normal power supply demand, and make the dispatching arrangement of the day through the capacity and quotation required by the load aggregator. 


\subsection{Participation Demand Response Analysis of Cluster Electric Heating Load}

\subsubsection{Electric Heating Load Participation Demand}

The response of electric power demand is mainly based on the price of electricity or encouragement measures, and change the original demand of electricity consumption to reduce or transfer the power consumption load of a certain time period, and eventually achieve scientific and reasonable power consumption, and ensure the stability of regional power grid. At present, the response based on the price demand is the core of current demand responses, the user can change the current demand of power consumption according to the price information of different periods, avoid the peak of power consumption as much as possible, or transfer to the low peak period ${ }^{[11]}$; the response of power demand is based on the supply and demand side, and the demand side power users can regulate the power demand according to the grid conditions, which promotes the interaction between the power company and the demand side users, improves the demand flexibility, and also realizes peak cutting and valley filling, and promotes the development of intermittent new energy resources.

\subsubsection{Characteristics of User Residents}

A case study on Jilin, a province in northern China, was performed. As people's living standards improve, the household appliances needed in daily life are increasing, including high-power electromagnetic heating and air conditioning, and the proportion of residents is constantly increasing. The electric heating load itself has a considerable high response speed and regulation ability; especially, during the heating period, it can maintain the indoor temperature within a comfort range. Chinese residents pay more and more attention to response resources on the demand side. The data show that in winter of 2019, the number of electric heating users in Jilin reached more than 3400 ; the electric heating can achieve low-carbon, and the heating area of clean electric energy in the same period has reached $254 \mathrm{~m} 2$. In the future, electric heating will become a considerably great demand response resource in the operation of power grids, and residents will be guided to join the power grid. Therefore, it has very important practical significance.

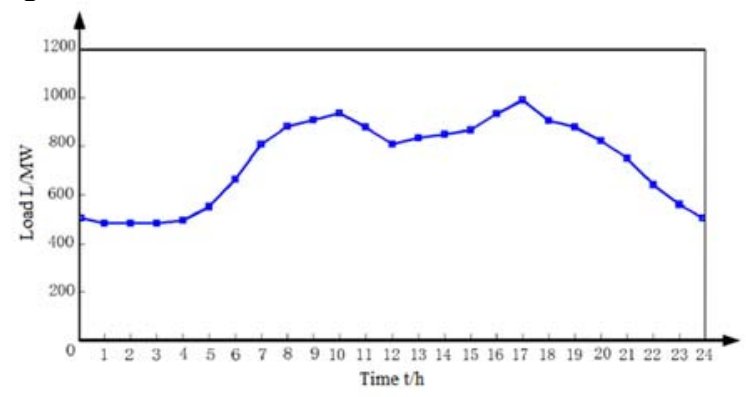

Fig. 5. Hourly Variation Curve of Daily Load in Winter of Jilin Province

\subsubsection{Electricity Price Mechanism in Response to Electricity Demand}

According to the price demand response mechanism, there are three kinds of electricity prices, which are timeof-use price, peak price, and real-time price. The timeof-time price mainly specifies different electricity prices according to the peak-demand period, normal period and low-demand period of electricity consumption. The electricity price in peak-demand period is the highest, while the electricity price in the low-demand period is the lowest to encourage users adjust the power using time and reach the goal of balancing the power needs across time. The peak price is an extreme value identified according to the price identified at the peakdemand period. Increasing the price can cut the peak demand and it can be used as the supplement of the time of use price. ${ }^{[12]}$ The real-time price is a pricing method that combines the long-term marginal cost with the short-term marginal cost under the premise of the instantaneous supply-demand balance and the safe operation of the power system. This method cannot only balance the power supply and demand in different time periods, but also realize the purpose of controlling the load curves through the price information and power users' rapid response. At the same time, it can promote the load changes due to the changes of real-time prices, and this kind of fluctuation can affect the prices in return. The specific performance is that when the user load increases, the corresponding electricity price will also increase, and vice versa. This way can reflect the flexible effect of users on electricity price.

\subsection{Participation Demand Response Analysis of Electric Heating Load Based on Real-time Electricity Price Model}

According to the demand response strategy of real-time electricity prices, the power demand of users will change according to the change of real-time electricity price in different time periods. Therefore, it can be assumed that the user load at time $T$ is $d(t)$, where $1 \leq t \leq 24$, the daily electricity consumption is $\mathrm{W}$, and the relationship between power load and residential electricity consumption is as follows:

$$
\mathrm{W}=\sum_{\mathrm{t}=1}^{24} d(t)
$$

If the real-time demand-based power pricing strategy is not adopted, the electricity price includes the power purchase cost, the output point loss, the transmission and distribution price and the government funds, so the total electricity cost is:

$$
\mathrm{Z}=\mathrm{Ca}+\mathrm{Cb}+\mathrm{Cc}+\mathrm{Cd}=\mathrm{PaW}+\mathrm{PcW}+\mathrm{PdW}
$$

where $\mathrm{Ca}, \mathrm{Cb}, \mathrm{Cc}$, and $\mathrm{Cd}$ in the formula represent the total power purchase cost, total bus loss cost, total transmission cost and the fundamental funds matched with the daily power consumption, respectively.

After implementing the corresponding strategy of electricity price, the performance of the elasticity of 
price for users is that the price will increase after the user load increases, and vice versa. Therefore, the electricity price level and real-time load level show positive correlation in different periods, so the proportion relationship between the real-time price and real-time load of the user in 24 hours can be shown as follows:

$$
P i: P j=d i: d j
$$

According to the proportion relationship, we can know that the real-time electricity price value of load ratio in period $i$ is $P_{i, t}$; therefore, we can get the following formula:

$$
P_{i, t}=\frac{d i}{\bar{d}} \times \frac{Z}{W}=\frac{24 \times d i}{\sum_{i=1}^{24} d i} \times\left(p_{a}+p_{b}+p_{c}+p_{d}\right)
$$

where $\overline{\mathrm{d}}$ is the average load of users in $24 \mathrm{~h}$.

\section{CONCLUSION}

As a good user demand response resource, the electric heating load can be regulated. The regulation strategies can reach multiple goals, and thus has seen wide adoption in engineering practice. In this paper, a thermal dynamic model was constructed based on the transient heat balance of electric heating, and the grouped electric heating demand response was explored.

\section{References}

1. Liu Yongkang, Lai Xiaoming, Gu Yibin, Wang Bo, Wang Guofeng, Liu Qing. Investigation on the ageing behaviour and hardening mechanisms of 5A90 Al-Li alloy U-shaped parts formed by electric resistance heating forming-quenching process[J]. Journal of Alloys and Compounds, 2021,869.

2. Schmitz N.,Sankowski L., Kaiser F.,Schwotzer C.,Echterhof T.,Pfeifer H.. Towards CO2-neutral process heat generation for continuous reheating furnaces in steel hot rolling mills - A case study[J]. Energy, 2021,224.

3. Zhao Zehui,Chen Huawei,Liu Xiaolin,Wang Zelinlan,Zhu Yantong,Zhou Yuping. The development of electric heating coating with temperature controlling capability for anti-icing/deicing $[\mathrm{J}]$. Cold Regions Science and Technology, 2021,184 .

4. Qu Xiaohang, Tian Maocheng. Flow-induced oscillation of membrane tapes and its effect on heat transfer of a transitional pipe flow[J]. International Journal of Heat and Mass Transfer,2021,171.

5. Zhao Ermeng, Hou Jian, Du Qingjun, Liu Yongge, Ji Yunkai, Bai Yajie. Numerical modeling of gas production from methane hydrate deposits using low-frequency electrical heating assisted depressurization method[J]. Fuel,2021,290.

6. Liu Kai, Xu Peixin, Wang Fang, Jin Can, Liu Quantao, Pang Huanping, Xie Hongzhou. The accumulated stress damage and residual life prediction of unreinforced concrete pavement with electric heating pipes[J]. Construction and Building Materials, 2021,278.

7. Gupta Ruchi, Pena-Bello Alejandro, Streicher Kai Nino, Roduner Cattia, Thöni David, Patel Martin Kumar, Parra David. Spatial analysis of distribution grid capacity and costs to enable massive deployment of PV, electric mobility and electric heating[J]. Applied Energy,2021,287.

8. Zhong Shengyuan, Wang Xiaoyuan, Zhao Jun, Li Wenjia, Li Hao, Wang Yongzhen, Deng Shuai, Zhu Jiebei. Deep reinforcement learning framework for dynamic pricing demand response of regenerative electric heating[J]. Applied Energy,2021,288.

9. Yu Meng, Li Sheng, Zhang Xuejun, Zhao Yang. Techno-economic analysis of air source heat pump combined with latent thermal energy storage applied for space heating in China[J]. Applied Thermal Engineering,2021,185.

10. Yong Zeng, Yutong Zeng, Dong Jiang, Shanhong Liu. Study on early-age thermal field of single-cell box girder concrete cured with electric heating furnaces[J]. Journal of Thermal Analysis and Calorimetry,2021(prepublish).

11. Sun Jian, Ma Shicai, Huo Cheng, Ge Zhihua, Zhou Shaoxiang. A Large Temperature Difference Thermal Substation (LTDTS) with Electric Heat Pump and Thermal Storage Tank[J]. IOP Conference Series: Earth and Environmental Science,2021,661(1).

12. Ximing Cheng, Yu Tang, Zhenpo Wang. Thermal Property Measurements of a Large Prismatic Lithium-ion Battery for Electric Vehicles[J]. Journal of Thermal Science,2021(prepublish).. 